\title{
Interactive comment on "Sensitivity of liquid cloud optical thickness and effective radius retrievals to cloud bow and glory conditions using two SEVIRI imagers" by Nikos Benas et al.
}

\section{Anonymous Referee \#2}

Received and published: 14 February 2019

Cloud properties retrieved from data of geostationary satellite sensors are largely employed by the scientific community. However, retrieval failures, i.e., the pixels implicitly excluded from the retrieval population could lead to biased estimates. Thus, it is of upmost importance to quantify failures, understand their sources and, if possible, develop algorithms of reprocessing of excluded pixels. Retrieval failures arise for various reasons. Some failure types were addressed in the cited in the manuscript literature. The work under reviewing is devoted to observation conditions favorable to cloud bow or cloud glory. The main idea of the work is to compare retrievals from the same type of data (SEVIRI reflectance) and for common areas but under different viewing an- 
addresses relevant scientific questions it is within the scope of AMT. I recommend that the paper be published in AMT after minor revisions.

AMTD

Specific comments:

Figures 6 and 11 show phase functions at intervals of angles of cloud bow and glory. It is seen that the phase functions are very sensitive to the effective size and to the effective variance. The reflectance of a cloud as measured from satellite at the topof-atmosphere should be less sensitive because of multiple scattering. It would be instructive to provide figures of angular dependence of the reflectance as additional figures along with Figs 6 and 11. Additional figures can be done for one typical value of the cloud optical thickness, say 8 , using the LUTs created by the authors. One observation geometry, say MSG-3 and the region west of the African coast, will be enough. Some of the Sun-satellite geometry angles can be constant and correspond to the case of March 7,2017 . The geometry angles should vary so that the intervals of scattering angles correspond to the intervals angles of Figs 6 and 11.

Figures 12 and 13 show retrievals results for $0.6 \mu \mathrm{m}-1.6 \mu \mathrm{m}$ channels and $0.6 \mu \mathrm{m}$ - $3.9 \mu \mathrm{m}$ channels separately. There is difference in the retrieved values, especially for the effective size. Sensitivity to the effective variance is seen as well. The authors should discuss how those properties can be used to estimate the effective variance.

Technical corrections.

Page 3 line 16 Replace Mayer et al. (2015) by Mayer et al. (2004).

The dotted and dashed lines are hardly distinguished in figures. I would prefer to see straight vertical lines of different color.

Interactive comment on Atmos. Meas. Tech. Discuss., doi:10.5194/amt-2018-439, 2019. 